\title{
Conformal wavefunctions for graviton amplitudes
}

\author{
Chang Liu and David A. Lowe \\ Department of Physics, Brown University, \\ Providence, RI 02912, U.S.A. \\ E-mail: chang_liu3@brown.edu, lowe@brown.edu
}

ABSTRACT: The extended-BMS algebra of asymptotically flat spacetime contains an $\mathrm{SO}(3,1)$ subgroup that acts by conformal transformations on the celestial sphere. It is of interest to study the representations of this subgroup associated with gravitons. To reduce the equation of motion to a Schrodinger-like equation it is necessary to impose a non-covariant gauge condition. Using these solutions, leading-order gauge invariant Weyl scalars are then computed and decomposed into families of unitary principal series representations. An invertible holographic mapping is constructed between these unitary principal series operators and massless spin-2 perturbations of flat spacetime.

KeYwords: Gauge-Gravity Correspondence, Models of Quantum Gravity, Space-Time Symmetries

ArXiv ePrint: 2109.00037 


\section{Contents}

1 Introduction 1

2 Graviton wavefunctions 2

2.1 Scalar perturbations 2

2.2 Vector perturbations 3

3 Klein-Gordon inner product $\quad 4$

3.1 Scalar perturbations 4

3.2 Vector perturbations 5

4 Gauge invariant observables 5

5 Holographic mapping to the celestial sphere $\quad 7$

6 Discussion 10

$\begin{array}{ll}\text { A Weyl scalars for scalar perturbation } & 11\end{array}$

$\begin{array}{ll}\text { B Weyl scalars for vector perturbation } & 11\end{array}$

\section{Introduction}

In previous work [1], massive scalar fields in 4D Minkowski spacetime were decomposed into modes on 3D de-Sitter spacetime slices where they form unitary principal series representations of $\mathrm{SO}(3,1)$. This study was motivated by the program of [2] where the goal is to formulate gravity in asymptotically flat spacetime as a theory on the celestial sphere with conformal symmetry. In this paper we extend this construction to massless spin-2 particles, or gravitons, in 4D Minkowski spacetime. To this end, we consider linearized gravitational waves living in flat $4 \mathrm{D}$ background spacetime with the standard spherical coordinates. The background metric is simply

$$
g_{\mu \nu}=\operatorname{diag}\left(-1,1, r^{2}, r^{2} \sin ^{2} \theta\right)
$$

Following the notations in [3], from now on indices $a, b, c, \ldots$ refer to the "orbit" spacetime labeled by the coordinates $(t, r)$, and $i, j, k, \ldots$ refer to the 2 -sphere labeled by $(\theta, \varphi)$. In other words, we write the background metric as

$$
g_{\mu \nu} \mathrm{d} x^{\mu} \mathrm{d} x^{\nu}=g_{a b} \mathrm{~d} y^{a} \mathrm{~d} y^{b}+r^{2} \mathrm{~d} \sigma^{2}
$$

where $g_{a b}=\operatorname{diag}(-1,1)$ and $\mathrm{d} \sigma^{2}=\gamma_{i j} \mathrm{~d} z^{i} \mathrm{~d} z^{j}=\mathrm{d} \theta^{2}+\sin ^{2} \theta \mathrm{d} \varphi^{2}$. 
For Minkowski spacetime in four dimensions, the gravitational perturbations can be expanded in terms of both the scalar and the vector spherical harmonics defined on the 2 -sphere. These are also known as the "even" and "odd" waves in [4], and will automatically have the desired transformation properties under the rotation group $\mathrm{SO}(3)$. However, as we shall see, the specific gauge conditions that we will choose in what follows are not Lorentz covariant, and therefore these metric perturbations do not transform as tensors under the full $\mathrm{SO}(3,1)$ group. To remedy this, we consider the Newman-Penrose formalism [5] of general relativity and construct leading-order gauge-invariant scalars known as the Weyl scalars. These scalars can then be mapped onto the celestial sphere through a generalization of the method described in the previous work [1]. This generalization involves performing a spectral decomposition into radial eigenvalues using the Meijer Ktransform [6, 7]. We therefore obtain an invertible holographic map between graviton fields on the flat Minkowski background spacetime and conformal operators on the celestial sphere. We stress that this procedure is defined for tree-level amplitudes and it remains to be seen whether an interacting holographic theory can be defined independently of the four-dimensional gravitational description.

\section{Graviton wavefunctions}

\subsection{Scalar perturbations}

Refs. [8, 9] present a general formalism which expresses the metric perturbation $h_{\mu \nu}$ in terms of a master function $\phi$. For the scalar perturbation, a gauge choice allows us to express the metric perturbation $h_{\mu \nu}$, expanded in terms of the spherical harmonics $Y_{l m}$, as

$$
h_{a b}=f_{a b} Y_{l m}, \quad h_{a i}=0, \quad h_{i j}=f \gamma_{i j} Y_{l m}
$$

where $f_{a b}$ and $f$ are functions that are related to a master function $\phi(t, r)$

$$
\begin{aligned}
f & =\frac{l(l+1) r \phi}{2}+r^{2} \partial_{r} \phi \\
f_{a b} & =\partial_{a} \partial_{b}(r \phi)-\frac{g_{a b}}{2} \square(r \phi) \\
& =r \partial_{a} \partial_{b} \phi+\partial_{a} r \partial_{b} \phi+\partial_{b} r \partial_{a} \phi-\frac{g_{a b}}{2}\left(r \square \phi+2 \partial_{r} \phi\right) .
\end{aligned}
$$

Here $\partial_{a} r \partial_{b} \phi$ stands for $\left(\partial_{a} r\right)\left(\partial_{b} \phi\right)$ and $\square$ is the d'Alembertian on the orbit spacetime. The components of $f_{a b}$ are

$$
\begin{aligned}
& f_{t t}=f_{r r}=r \partial_{t}^{2} \phi+\partial_{r} \phi+\frac{l(l+1)}{2 r} \phi \\
& f_{t r}=r \partial_{t} \partial_{r} \phi+\partial_{t} \phi .
\end{aligned}
$$

The master function $\phi(t, r)$ satisfies the following master equation

$$
\square \phi-\frac{l(l+1)}{r^{2}} \phi=0
$$


which can be solved to yield a basis of mode functions

$$
\phi_{\omega l}(t, r)=e^{-i \omega t} \sqrt{r}\left(c_{1} J_{l+\frac{1}{2}}(\omega r)+c_{2} Y_{l+\frac{1}{2}}(\omega r)\right)
$$

for $\omega \neq 0$. Here $J_{n}$ is the Bessel function of first kind and $Y_{n}$ is the Bessel function of second kind. We demand that the mode functions be regular at the origin, and therefore discard the second set of solutions. We therefore find the (unnormalized) modes for the master function

$$
\phi_{\omega l}(t, r)=e^{-i \omega t} \sqrt{r} J_{l+\frac{1}{2}}(\omega r) .
$$

For $\omega \neq 0$ this agrees with the master equation found in ref. [10].

For $\omega=0$ we have the special time-independent solution of the master equation

$$
\phi_{0 l}(t, r)=c_{1} r^{l+1}+c_{2} r^{-l}
$$

Discarding solutions that are divergent at $r \rightarrow \infty$ we find the basis of functions for the $\omega=0$ modes

$$
\phi_{0 l}(t, r)=r^{-l}
$$

Physically this represents a time-independent spacetime perturbation that is rotating at a constant angular momentum (for $l \neq 0$. For $l=0$ the metric perturbation is zero), similar to the eternal Kerr black hole which appears to a distant observer as having a total angular momentum. Substituting the master function into eq. (2.1) we find the scalar metric perturbation for $\omega=0$

$$
h_{\mu \nu}=Y_{l m}\left[\begin{array}{cccc}
\frac{l(l-1)}{2} r^{-l-1} & 0 & 0 & 0 \\
0 & \frac{l(l-1)}{2} r^{-l-1} & 0 & 0 \\
0 & 0 & \frac{l(l-1)}{2} r^{1-l} & 0 \\
0 & 0 & 0 & \frac{l(l-1)}{2} r^{1-l} \sin ^{2} \theta
\end{array}\right] .
$$

\subsection{Vector perturbations}

We define the vector spherical harmonics as a vector field $Y_{i}^{(l m)}$ on the unit 2-sphere satisfying

$$
\left[\Delta_{2}+(l(l+1)-1)\right] Y_{i}^{(l m)}=0
$$

with $D^{i} Y^{(l m)}{ }_{i}=0$. Here $\Delta_{2}$ and $D_{i}$ are the Laplace operator and the covariant derivative on the unit 2-sphere, respectively. In terms of the scalar spherical harmonics $Y_{l m}$ we find

$$
Y_{i}^{(l m)}(\theta, \varphi)=\frac{\epsilon_{i j}}{\sqrt{l(l+1)}} \partial^{j} Y_{l m}(\theta, \varphi)
$$

Here indices are raised and lowered using the metric $\gamma_{i j}$ on the unit 2-sphere, and $\epsilon^{i j}$ is the Levi-Civita tensor on the 2-sphere defined by $\epsilon_{\theta \varphi}=\sqrt{\left|\operatorname{det} \gamma_{i j}\right|}=\sin \theta$. Given suitable gauge choice [4], the metric perturbation can then be expanded in terms of $Y_{i}^{(l m)}$ as follows

$$
h_{a b}=0, \quad h_{a i}=f_{a} Y_{i}^{(l m)}, \quad h_{i j}=0
$$


where $f^{a}$ is related to the master function $\phi(t, r)$ above via

$$
f^{a}=\epsilon^{a b} \partial_{b}(r \phi)
$$

Here $\epsilon_{a b}$ is the Levi-Civita tensor on the two-dimensional orbit spacetime defined by $\epsilon^{t r}=$ +1 . For $\omega \neq 0$ this agrees with the so-called odd waves in ref. [4]. For $\omega=0$ substituting the master function $\phi=r^{-l}$ into eq. (2.3) we find the metric perturbation

$$
h_{\mu \nu}=\sqrt{\frac{l}{l+1}} r^{-l-1}\left[\begin{array}{cccc}
0 & 0 & \frac{1}{\sin \theta} \partial_{\varphi} Y_{l m}-\sin \theta \partial_{\theta} Y_{l m} \\
0 & 0 & 0 & 0 \\
\frac{1}{\sin \theta} \partial_{\varphi} Y_{l m} & 0 & 0 & 0 \\
-\sin \theta \partial_{\theta} Y_{l m} & 0 & 0 & 0
\end{array}\right] .
$$

\section{Klein-Gordon inner product}

The metric perturbation expressions constructed above are yet to be normalized. Following [9] we define the inner product between two metric perturbations $h_{\mu \nu}$ and $h_{\mu \nu}^{\prime}$ as

$$
\left\langle h, h^{\prime}\right\rangle=-i \int_{\Sigma} \mathrm{d} \Sigma n_{\lambda}\left(h_{\mu \nu}^{\star} p^{\prime \lambda \mu \nu}-h_{\mu \nu}^{\prime} p^{\star \lambda \mu \nu}\right)
$$

where $\Sigma$ is a Cauchy surface and $n^{\lambda}$ is the future-directed unit vector field normal to $\Sigma$. Here $p^{\lambda \mu \nu}$ is the conjugate momentum current

$$
p^{\lambda \mu \nu}=g^{\lambda \nu} \nabla_{\kappa} h^{\kappa \nu}+g^{\lambda \nu} \nabla_{\kappa} h^{\kappa \mu}-\nabla^{\lambda} h^{\mu \nu}+g^{\mu \nu}\left(\nabla^{\lambda} h-\nabla^{\kappa} h^{\lambda}{ }_{\kappa}\right)-\frac{g^{\lambda \nu} \nabla^{\mu} h+g^{\lambda \mu} \nabla^{\nu} h}{2}
$$

Here all indices are raised and lowered with respect to the background metric $g_{\mu \nu}$ and $\nabla$ is the covariant derivative of the background metric.

\subsection{Scalar perturbations}

For the scalar perturbation ref. [9] eq. 88 has shown that for $h_{\mu \nu}^{\omega l m}$ and $h_{\mu \nu}^{\omega^{\prime} l^{\prime} m^{\prime}}$, derived from the master function $\phi_{\omega l}$ and $\phi_{\omega^{\prime} l^{\prime}}$ via eq. (2.1) respectively, the conserved inner product is

$$
\left\langle h^{\omega l m}, h^{\omega^{\prime} l^{\prime} m^{\prime}}\right\rangle=-i \int_{0}^{+\infty} \mathrm{d} r \delta_{l l^{\prime}} \delta_{m m^{\prime}} J^{0}
$$

where the orbit spacetime current $J^{a}$ is given by

$$
J^{a}=\frac{4}{r} \partial^{c} r\left(f^{\star a b} f_{b c}^{\prime}-f^{a b} f_{b c}^{\prime \star}\right)-\left(f^{\star b c} \partial^{a} f_{b c}^{\prime}-f^{\prime b c} \partial^{a} f_{b c}^{\star}\right)
$$

Here $f_{a b}$ is related to the master function $\phi_{\omega l}$ and $f_{a b}^{\prime}$ is related to the master function $\phi_{\omega^{\prime} l^{\prime}}$ via eq. (2.2). Substituting eq. (2.2) and following the derivations in eqs. 90-100 and appendix B of ref. [9] we find

$$
J^{0}=-\frac{l(l-1)(l+1)(l+2)}{2}\left(\phi_{\omega l}^{\star} \partial_{t} \phi_{\omega^{\prime} l^{\prime}}-\phi_{\omega^{\prime} l^{\prime}} \partial_{t} \phi_{\omega l}^{\star}\right) .
$$


For $\phi_{\omega l}=e^{-i \omega t} \sqrt{r} J_{l+\frac{1}{2}}(\omega r)$ we have

$$
\left.i\left(\phi_{\omega l}^{\star} \partial_{t} \phi_{\omega^{\prime} l}-\phi_{\omega^{\prime} l} \partial_{t} \phi_{\omega l}^{\star}\right)\right|_{t=0}=\left(\omega+\omega^{\prime}\right) r J_{l+\frac{1}{2}}(r \omega) J_{l+\frac{1}{2}}\left(r \omega^{\prime}\right) \text {. }
$$

Using

$$
\int_{0}^{\infty} r J_{\alpha}(\omega r) J_{\alpha}\left(\omega^{\prime} r\right) \mathrm{d} r=\frac{\delta\left(w-w^{\prime}\right)}{\omega}
$$

we find

$$
\left\langle h^{\omega l m}, h^{\omega^{\prime} l^{\prime} m^{\prime}}\right\rangle=l(l-1)(l+1)(l+2) \delta\left(\omega-\omega^{\prime}\right) \delta_{l l^{\prime}} \delta_{m m^{\prime}} .
$$

We see that in order to normalize the metric perturbations to have

$$
\left\langle h^{\omega l m}, h^{\omega^{\prime} l^{\prime} m^{\prime}}\right\rangle=\delta\left(\omega-\omega^{\prime}\right) \delta_{l l^{\prime}} \delta_{m m^{\prime}}
$$

we will perform a change of variable $h_{\mu \nu} \rightarrow[l(l-1)(l+1)(l+2)]^{-1 / 2} h_{\mu \nu}$. In the interest of notational clarity we will assume this has been done and will continue to use $h_{\mu \nu}$ to denote the normalized scalar metric perturbation.

\subsection{Vector perturbations}

For the vector perturbations ref. [9] eq. A10 has shown that for $h_{\mu \nu}^{V}$ and $h^{\prime}{ }_{\mu \nu}$ given by master functions $\phi_{\omega l m}$ and $\phi_{\omega^{\prime} l^{\prime} m^{\prime}}$ (via eq. (2.3)) we have

$$
\left\langle h^{V}, h^{\prime V}\right\rangle=-i \delta_{l l^{\prime}} \delta_{m m^{\prime}}(l-1)(l+2) \int_{0}^{+\infty} \mathrm{d} r\left(\phi_{\omega l}^{\star} \partial_{t} \phi_{\omega^{\prime} l^{\prime}}-\phi_{\omega^{\prime} l^{\prime}} \partial_{t} \phi_{\omega l}^{\star}\right) .
$$

Following the calculations of the previous section we find

$$
\left\langle h^{V}, \tilde{h}^{V}\right\rangle=2(l-1)(l+2) \delta\left(\omega-\omega^{\prime}\right) \delta_{l l^{\prime}} \delta_{m m^{\prime}} .
$$

We see that a change of variable $h_{\mu \nu}^{V} \rightarrow[2(l-1)(l+2)]^{-1 / 2} h_{\mu \nu}$ will allow us to normalize the vector metric perturbations to have

$$
\left\langle\left(h^{V}\right)^{\omega l m},\left(h^{V}\right)^{\omega^{\prime} l^{\prime} m^{\prime}}\right\rangle=\delta\left(\omega-\omega^{\prime}\right) \delta_{l l^{\prime}} \delta_{m m^{\prime}} .
$$

\section{Gauge invariant observables}

The solutions we have described above depend on the choice of gauge (2.1). Since our graviton modes are expanded in terms of scalar or vector spherical harmonics, we expect that they transform under the rotation group $\mathrm{SO}(3)$ in the usual way. Indeed, denoting $\left(h^{S}\right)_{\mu \nu}^{\omega l m}$ by $|\omega l m\rangle$, we find that

$$
L_{3}|\omega l m\rangle=m|\omega l m\rangle
$$

and

$$
\left(L_{1} \pm i L_{2}\right)|\omega l m\rangle=\sqrt{(l \mp m)(l \pm m+1)}|\omega l, m \pm 1\rangle
$$

where the rotation operators $L_{i}$ act on the graviton modes as

$$
L_{i} h_{\mu \nu}=-i\left[\epsilon_{i j k} x^{j} \partial^{k} h_{\mu \nu}+\left(\omega_{i j}\right)_{\mu}{ }^{\lambda} h_{\lambda \nu}+\left(\omega_{i j}\right)_{\nu}{ }^{\lambda} h_{\mu \lambda}\right]
$$


with

$$
\left(\omega_{\mu \nu}\right)_{\alpha}^{\beta}=\eta_{\mu \alpha} \delta_{\nu}^{\beta}-\eta_{\nu \alpha} \delta_{\mu}^{\beta} .
$$

However, since the gauge conditions (eqs. (2.1), (2.3)) for either the scalar and vector perturbations are not Lorentz covariant, we do not expect that these graviton modes transform as representations of the full Lorentz group $\mathrm{SO}(3,1)$. Indeed, acting with the boost operators

$$
K_{i} h_{\mu \nu}=-i\left[\left(x^{0} \partial^{i}-x^{i} \partial^{0}\right) h_{\mu \nu}+\left(\omega_{0 i}\right)_{\mu}{ }^{\lambda} h_{\lambda \nu}+\left(\omega_{0 i}\right)_{\nu}{ }^{\lambda} h_{\mu \lambda}\right]
$$

does not produce the correct Lorentz algebra. In order to restore the correct Lorentz algebra, one needs to perform additional gauge transformations after a boost to restore the gauge conditions (eqs. (2.1), (2.3)). The exact gauge transformations required are non-trivial and do not have a closed expression as far as we know.

Alternatively, one could consider the transverse-traceless gauge that is indeed Lorentz covariant and rewrite our graviton modes in this gauge, and attempt to quantize such a theory with a procedure similar to the Gupta-Bleuler formalism of electromagnetism. However this procedure does not provide a complete gauge fixing, and one is still left with a Hilbert space containing zero-norm states. Neither of these approaches will be completely satisfactory in producing a conformal description of the graviton modes as gauge invariant operators on the celestial sphere.

In the context of AdS/CFT the usual procedure would be to adopt Fefferman-Graham coordinates where one can simply identify components of the metric expansion around spatial infinity with a boundary stress-energy tensor. The boundary stress-energy tensor then provides a complete description of the boundary data for gravitational waves.

Finding an analogous set of variables in the case of asymptotically flat spacetime is a somewhat more thorny problem. One approach is simply to choose a Bondi metric near null infinity $[11,12]$ and describe the gravitational waves using the asymptotic variables that appear there. Another approach, used in the numerical study of gravitational waves from time-dependent collapsing/colliding objects, is to instead pick a distinguished tetrad and compute the so-called Weyl scalars (see for example [13, 14]). An infinitesimal gauge transformation of the Riemann tensor is

$$
\delta R_{\mu \nu \beta}^{\alpha}=\mathcal{L}_{\xi} R_{\mu \nu \beta}^{\alpha}
$$

where $\xi$ parameterizes the diffeomorphism and $\mathcal{L}_{\xi}$ is the Lie derivative. Since the Lie derivative is linear in $R_{\mu \nu \beta}^{\alpha}$ and linear in $\xi$ this will vanish at leading order in $\xi$ if the Riemann tensor is computed at linear order in the perturbation around flat spacetime. So the Weyl scalars, which amount to picking particular components of the Riemann tensor in this context, will be a set of gauge invariant observables at leading order.

We are therefore led to the consideration of the so-called spin-coefficient formalism [15] of general relativity, a special example of which is known as the Newman-Penrose formalism [5]. Here one picks a null tetrad satisfying

$$
l_{\mu} l^{\mu}=n_{\mu} n^{\mu}=m_{\mu} m^{\mu}=\bar{m}_{\mu} \bar{m}^{\mu}=0
$$


normalized so that

$$
l_{\mu} n^{\mu}=-1, \quad m_{\mu} \bar{m}^{\mu}=1
$$

with other cross contractions between two vectors vanishing. For our specific purpose we will pick the limit of the Kinnersley tetrad [16]

$$
\begin{aligned}
l^{\mu} & =(1,1,0,0), & n^{\mu} & =\left(\frac{1}{2},-\frac{1}{2}, 0,0\right), \\
m^{\mu} & =\frac{1}{\sqrt{2} r}\left(0,0,1, \frac{i}{\sin \theta}\right), & \bar{m}^{\mu} & =\frac{1}{\sqrt{2} r}\left(0,0,1,-\frac{i}{\sin \theta}\right) .
\end{aligned}
$$

The five Weyl scalars $\Psi_{A}$ for $A=0, \ldots, 4$ are built out of the Weyl tensor $C_{\alpha \beta \gamma \delta}$ of the full spacetime as

$$
\begin{aligned}
& \Psi_{0}=C_{\alpha \beta \gamma \delta} l^{\alpha} m^{\beta} l^{\gamma} m^{\delta} \\
& \Psi_{1}=C_{\alpha \beta \gamma \delta} l^{\alpha} n^{\beta} l^{\gamma} m^{\delta} \\
& \Psi_{2}=C_{\alpha \beta \gamma \delta} l^{\alpha} m^{\beta} \bar{m}^{\gamma} n^{\delta} \\
& \Psi_{3}=C_{\alpha \beta \gamma \delta} l^{\alpha} n^{\beta} \bar{m}^{\gamma} n^{\delta} \\
& \Psi_{4}=C_{\alpha \beta \gamma \delta} n^{\alpha} \bar{m}^{\beta} n^{\gamma} m^{\delta} .
\end{aligned}
$$

Here we expand $C_{\alpha \beta \gamma \delta}$ to first order in $h_{\mu \nu}$. Since the full spacetime satisfies the vacuum Einstein equation, we find that the Weyl tensor $C_{\alpha \beta \gamma \delta}$ is equal to the Riemann tensor $R_{\alpha \beta \gamma \delta}$. The Weyl scalars, gauge invariant under infinitesimal gauge transformations, may then be expressed in terms of the graviton wavefunctions of the previous section. Our strategy will then be to decompose these gauge invariant scalars into representations of the conformal group.

Note that for a particular value of $l, m$ it would be possible to find modification of the tetrad, at leading order in the perturbation, such as picking $l$ along a principal null direction, that would make some of the Weyl scalars vanish. However if make pick the tetrad independently of the perturbation, all the Weyl scalars will typically be non-vanishing, and will provide a basis for gauge invariant observables (at leading order).

A straightforward calculation leads to the expressions for the Weyl scalars evaluated on the scalar and vector perturbations in appendices A and B respectively. These expressions involve radial derivations of the master function $\phi(t, r)$ and angular derivatives of the spherical harmonics. For us, the main point is that the Weyl scalars do not satisfy a simple wave equation in $4 \mathrm{D}$ spacetime. In order to use the methods of [1] we next must perform a spectral decomposition in the radial direction to allow us to use that basis functions as a complete basis for the Weyl scalars.

\section{$5 \quad$ Holographic mapping to the celestial sphere}

The Weyl scalars encode all the information of the gravitational perturbations. Our strategy will be to proceed in two steps: first consider a fixed radius 3D de Sitter slice of flat spacetime, and use the Plancherel (or completeness) theorem for the unitary principle series 
representations to decompose a general function on such a slice into irreducible representations; next we allow for a general radial variation of such functions, effectively decomposing a general solution into solutions of the $4 \mathrm{D}$ massive scalar wave equations with a continuous spectrum of masses.

The starting point is the unitary principal series mode functions that we have computed in $[1]$

$$
\Phi_{p M l m}(\eta, \rho, z, \bar{z})=\phi_{p l}(\eta) \psi_{p M}(\rho) Y_{m}^{l}(z, \bar{z})
$$

where

$$
\phi_{p l}(\eta)=\operatorname{sech} \eta\left[\frac{i \pi}{2} P_{l}^{i \sqrt{p^{2}-1}}(\tanh \eta)+Q_{l}^{i \sqrt{p^{2}-1}}(\tanh \eta)\right]
$$

and

$$
\psi_{p M}(\rho)=\frac{K_{i \sqrt{p^{2}-1}}(M \rho)}{\rho} .
$$

Here $M$ is the 4 D scalar mass, $l, m$ are the usual angular momentum quantum numbers, and $p$ labels the unitary principal series representation and also behaves as a radial quantum number. $K_{\nu}(x)$ is the modified Bessel function of second kind, and $(\eta, \rho, z, \bar{z})$ are the hyperbolic coordinates on Minkowski spacetime with metric

$$
\mathrm{d} s^{2}=-\rho^{2} \mathrm{~d} \eta^{2}+\mathrm{d} \rho^{2}+\rho^{2} \cosh ^{2} \eta \frac{4 \mathrm{~d} z \mathrm{~d} \bar{z}}{\left(1+|z|^{2}\right)^{2}} .
$$

We will apply the main result of ref. [1], which states that these modes $\Phi_{p M l m}$ form a unitary principal series representation of $\mathrm{SO}(3,1)$. This allows us to apply the Plancherel theorem [17] and map the Weyl scalars into sets of conformal operators defined on the celestial sphere. We now discuss this procedure in detail.

To motivate the full 4D map, let us first consider the simpler case where one is to construct a holographic map on the 3D de-Sitter slice (which we take to be the $\rho=1$ hypersurface) of $4 \mathrm{D}$ Minkowski. On the $3 \mathrm{D}$ de-Sitter slice, the mode functions $\Phi_{p M l m}$ above reduce to the mode functions $\phi_{p l m}(\eta, z, \bar{z})=\phi_{p l}(\eta) Y_{l m}(z, \bar{z})$. We can then use these modes as basis for the analogue of Fourier transform, and maps a scalar function $f(\eta, z, \bar{z})$ into "Fourier" coefficients labeled by $\hat{f}(p, l, m)$

$$
\hat{f}(p, l, m)=\int_{-\infty}^{+\infty} \cosh ^{2} \eta \mathrm{d} \eta \int_{\mathbb{C}} \frac{4 \mathrm{~d} z \mathrm{~d} \bar{z}}{\left(1+|z|^{2}\right)^{2}} \bar{\phi}_{p l}(\eta) \bar{Y}_{l m}(z, \bar{z}) f(\eta, z, \bar{z}) .
$$

The Plancherel theorem [17] guarantees that this map is unitary. Using eq. 10.40 of ref. [17], we find the inverse map

$$
f(\eta, z, \bar{z})=\int_{1}^{\infty} 2 p \tanh \left(\frac{\pi}{2} \sqrt{p^{2}-1}\right) \mathrm{d} p \sum_{l m} \phi_{p l}(\eta) Y_{l m}(z, \bar{z}) \hat{f}(p, l, m) .
$$

In particular, the integral measure of the inverse map follows from setting $v=\sqrt{p^{2}-1}$ in $v \tanh (\pi v / 2) \mathrm{d} v$ of eq. 10.40 [17], and multiplying it by two, since both $p \in(1,+\infty)$ and $p \in(-\infty,-1)$ map to $v \in(0,+\infty)$. 
In order to include the radial variation of the scalars, we note the fact that the following Meijer $K$-transform of order $\nu$, defined for a function $f(x)$ as

$$
\hat{f}(y)=\int_{0}^{\infty} f(x) K_{\nu}(x y)(x y)^{1 / 2} \mathrm{~d} x
$$

has the following inverse transform

$$
f(x)=\frac{1}{\pi i} \int_{c-i \infty}^{c+i \infty} \hat{f}(y) I_{\nu}(x y)(x y)^{1 / 2} \mathrm{~d} y
$$

where $c$ is an arbitrary real number and $I_{\nu}(x)$ is the modified Bessel function of the first kind. This is developed in a series of papers $[6,7,18,19]$ and summarized in Chapter $\mathrm{X}$ of ref. [20], which we simply quote without going into further details of the proof. This gives us a unitary transformation for the radial component, from which we obtain the full 4D forward and inverse map: given a scalar function $\Psi(\eta, \rho, z, \bar{z})$, we define the following analogue of the Fourier transform on four-dimensional Minkowski spacetime

$$
\begin{aligned}
\hat{\Psi}(p, M, l, m)= & \int_{-\infty}^{+\infty} d \eta \bar{\phi}_{p l}(\eta) \cosh ^{2} \eta \int_{0}^{\infty} d \rho K_{i \sqrt{p^{2}-1}}(M \rho)(M \rho)^{1 / 2} \\
& \times \int_{\mathbb{C}} \frac{4 \mathrm{~d} z \mathrm{~d} \bar{z}}{\left(1+|z|^{2}\right)^{2}} \bar{Y}_{l m}(z, \bar{z}) \Psi(\eta, \rho, z, \bar{z})
\end{aligned}
$$

that maps any scalar function $\Psi(\eta, \rho, z, \bar{z})$ to "Fourier" coefficients $\hat{\Psi}$ labeled by $(p, M, l, m)$, where $p>1, M>0, l \geq 0$, and $-l \leq m \leq l$. We note this $\rho$ integral is indeed convergent. This transformation is invertible, with the inverse map given by

$$
\begin{aligned}
\Psi(\eta, \rho, z, \bar{z})= & \frac{1}{\pi i} \int_{1}^{+\infty} 2 p \tanh \left(\frac{\pi}{2} \sqrt{p^{2}-1}\right) \mathrm{d} p \int_{-i \infty}^{+i \infty} I_{i \sqrt{p^{2}-1}}(M \rho)(M \rho)^{1 / 2} \mathrm{~d} M \\
& \times \sum_{l=0}^{\infty} \sum_{m=-l}^{l} \phi_{p l}(\eta) Y_{l m}(z, \bar{z}) \hat{\Psi}(p, M, l, m)
\end{aligned}
$$

Here $I_{\nu}(x)$ is the modified Bessel function of the first kind.

We are now in a position to apply the forward and inverse map described above to each of the Weyl scalars of the gravitational modes computed earlier. This allows us to identify two families of celestial sphere operators that encode the 5 complex Weyl scalars for each variety of perturbation (scalar and vector) which we can label as $\hat{\Psi}_{p M l m}^{S, A}$ and $\hat{\Psi}_{p M l m}^{V, A}$. Here $S, V$ refer to scalar and vector, and $A=0, \cdots, 4$ labels the 5 Weyl scalars. The $l, m$ angular momentum space is conjugate to the 2 -sphere coordinate space $z, \bar{z}$ so in this sense we obtain a holographic mapping of the gravitational modes to the celestial sphere.

These celestial sphere operators will automatically have the desired conformal transformation properties as shown in [1]. We therefore find that the procedure described above allows us to build a celestial sphere description of the gravitational modes living in the Minkowski bulk spacetime at leading order. The modes are expressed as linear combinations of unitary irreducible representations of the conformal group acting on the celestial sphere. 


\section{Discussion}

We have constructed a holographic map between gravitational perturbations around a Minkowski background and conformal operators in the 2D boundary known as the celestial sphere. The forward map, going from a metric perturbation $g_{\mu \nu}=\eta_{\mu \nu}+h_{\mu \nu}$ in $4 \mathrm{D}$ to conformal operators $\hat{\Psi}_{p M l m}^{A}$ in 2D celestial sphere, is the following:

1. Compute the Riemann tensor $R_{\alpha \beta \gamma \delta}$ to first order in $h_{\mu \nu}$. Since the full spacetime satisfies the vacuum Einstein equation, the Weyl tensor $C_{\alpha \beta \gamma \delta}$ is equal to the Riemann tensor $R_{\alpha \beta \gamma \delta}$

2. From the Weyl tensor, compute the five Weyl scalars $\Psi^{A}$ using (4.1) and (4.2).

3. Apply the forward map (5.1) to the five Weyl scalars, and obtain five conformal operators $\hat{\Psi}_{p M l m}^{A}$ on the celestial sphere, each labeled by four quantum numbers $p$, $M, l$, and $m$.

Likewise, the inverse map, starting from the complete set of conformal operators $\hat{\Psi}_{p M l m}^{A}$, is to apply (5.2) to obtain the five (complex) Weyl scalars $\Psi^{A}(\eta, \rho, z, \bar{z})$. These five complex Weyl scalars encode the ten independent components of the Weyl tensor, which fully specify the metric perturbation $h_{\mu \nu}$, up to coordinate (gauge) choices. In particular, one can apply the holographic map above to the case of individual gravitational wave modes (there are two types, scalar and vector perturbations, see (2.1)-(2.2) and (2.3)) and obtain 2D conformal operators on the celestial sphere that describe $4 \mathrm{D}$ gravitational perturbations around flat spacetime.

We stress that this procedure uses kinematic information to simply organize the modes in a convenient way. With interactions included, one can still use this procedure to map bulk dynamics to the celestial sphere. The important question then is whether the dynamics has any useful description incorporating the conformal symmetry of the celestial sphere as in the program advocated in [2]. In the case of AdS/CFT the analogous answer was the holographic theory was simpler than the gravity theory, being a quantum field theory with conformal symmetry. In the case of asymptotically flat spacetime it remains unclear whether the celestial sphere theory is a quantum field theory. It remains a logical possibility that the 4D gravitational description will be the simplest way to describe the full dynamics of the theory. Another approach is to take AdS/CFT in the limit of vanishing cosmological constant where shock waves can be included to encode nontrivial BMS charges, and the holographic description involves a 3D CFT with boundary [21]. In any case, the work presented here shows how to decompose any such description into the underlying operator content living on the celestial sphere and transforming simply under conformal transformations. This is a necessary first step in constructing complete holographic descriptions of asymptotically flat spacetime.

\section{Acknowledgments}

C.L. and D.L. are supported in part by DOE grant de-sc0010010 Task A. 


\section{A Weyl scalars for scalar perturbation}

The Weyl scalars are evaluated at linear order for the scalar perturbation mode $h_{\mu \nu}^{\omega l m}$ defined above. Here we use $\phi^{\prime}=\frac{\partial}{\partial r} \phi$.

$$
\begin{aligned}
& \Psi_{0}=-\frac{1}{4 r^{3} \sqrt{\Gamma(l-m-1)} \sqrt{\Gamma(l-m)} \sqrt{\Gamma(l+m+1)}}\left(\phi\left(l^{2}+l-2 r \omega(r \omega+i)\right)+2 r(1-i r \omega) \phi^{\prime}\right) \\
& \times\left(\sqrt { \Gamma ( l - m ) } \left(\sqrt{\Gamma(l-m+1)} \sqrt{\Gamma(l+m+3)} e^{-i 2 \varphi} Y_{l}^{m+2}\right.\right. \\
& \left.+(m-1) m \tan ^{2}\left(\frac{\theta}{2}\right) \sqrt{\Gamma(l-m-1)} \sqrt{\Gamma(l+m+1)} Y_{l}^{m}\right) \\
& \left.-2 m e^{-i \varphi} \tan \left(\frac{\theta}{2}\right) \sqrt{\Gamma(l-m-1)} \sqrt{\Gamma(l-m+1)} \sqrt{\Gamma(l+m+2)} Y_{l}^{m+1}\right) \\
& \Psi_{1}=\frac{1}{4 \sqrt{2} r^{3} \sqrt{\Gamma(l-m)} \sqrt{\Gamma(l+m+1)}} e^{-i \varphi}\left(r\left(\left(l^{2}+l-2\right) \phi^{\prime}+2 r(1-i r \omega) \phi^{\prime \prime}\right)\right. \\
& \left.+i \phi\left(\left(l^{2}+l+2\right) r \omega+2 i l(l+1)-2 r^{3} \omega^{3}-2 i r^{2} \omega^{2}\right)\right) \\
& \times\left(\sqrt{\Gamma(l-m+1)} \sqrt{\Gamma(l+m+2)} Y_{l}^{m+1}-m e^{i \varphi} \tan \left(\frac{\theta}{2}\right) \sqrt{\Gamma(l-m)} \sqrt{\Gamma(l+m+1)} Y_{l}^{m}\right) \\
& \Psi_{2}=-\frac{1}{8 r^{3}} Y_{l}^{m}\left(r\left(r\left(l(l+1) \phi^{\prime \prime}+2 r \phi^{\prime \prime \prime}\right)-2\left(l^{2}+l-r^{2} \omega^{2}\right) \phi^{\prime}\right)+l(l+1) \phi\left(r^{2} \omega^{2}+2\right)\right) \\
& \Psi_{3}=\frac{1}{8 \sqrt{2} r^{3} \sqrt{\Gamma(l-m)} \sqrt{\Gamma(l+m+1)}} e^{-i \varphi}\left(r\left(-\left(l^{2}+l-2\right) \phi^{\prime}+2 r(-1-i r \omega) \phi^{\prime \prime}\right)\right. \\
& \left.+\phi\left(i\left(l^{2}+l+2\right) r \omega+2 l(l+1)-2 i r^{3} \omega^{3}-2 r^{2} \omega^{2}\right)\right) \\
& \times\left(\sqrt{\Gamma(l-m+1)} \sqrt{\Gamma(l+m+2)} Y_{l}^{m+1}+m e^{i \varphi} \cot \left(\frac{\theta}{2}\right) \sqrt{\Gamma(l-m)} \sqrt{\Gamma(l+m+1)} Y_{l}^{m}\right) \\
& \Psi_{4}=-\frac{1}{16 r^{3} \sqrt{\Gamma(l-m-1)} \sqrt{\Gamma(l-m)} \sqrt{\Gamma(l+m+1)}} e^{-i 2 \varphi}\left(\phi\left(l^{2}+l-2 r \omega(r \omega-i)\right)+2 r(1+i r \omega) \phi^{\prime}\right) \\
& \times\left(\sqrt{\Gamma(l-m)} \sqrt{\Gamma(l-m+1)} \sqrt{\Gamma(l+m+3)} Y_{l}^{m+2}\right. \\
& +m e^{i \varphi} \cot \left(\frac{\theta}{2}\right) \sqrt{\Gamma(l-m-1)}\left(2 \sqrt{\Gamma(l-m+1)} \sqrt{\Gamma(l+m+2)} Y_{l}^{m+1}\right. \\
& \left.\left.+(m-1) e^{i \varphi} \cot \left(\frac{\theta}{2}\right) \sqrt{\Gamma(l-m)} \sqrt{\Gamma(l+m+1)} Y_{l}^{m}\right)\right) .
\end{aligned}
$$

\section{B Weyl scalars for vector perturbation}

The Weyl scalars are evaluated at linear order for the vector perturbation mode $h_{\mu \nu}^{\omega l m}$ defined above. Here we use $\phi^{\prime}=\frac{\partial}{\partial r} \phi$. For brevity of presentation we follow [4] and present the $m=0$ modes. The $m \neq 0$ modes can always be obtained by acting with an $\mathrm{SO}(3)$ 
generator.

$$
\begin{aligned}
\Psi_{0}= & \frac{\sqrt{\Gamma(l+3)} e^{-i 2 \varphi} Y_{l}^{2}\left(i r \phi^{\prime \prime}+2(r \omega+i) \phi^{\prime}+\omega \phi(2-i r \omega)\right)}{2 \sqrt{l(l+1)} r^{2} \sqrt{\Gamma(l-1)}} \\
\Psi_{1}= & -\frac{\sqrt{\Gamma(l+2)} e^{-i \varphi} Y_{l}^{1}}{2 \sqrt{2} \sqrt{l(l+1)} r^{2} \sqrt{\Gamma(l)}} \\
& \times \frac{\left(i\left(\left(r^{2} \omega^{2}-2\right) \phi^{\prime}+r\left(r \phi^{\prime \prime \prime}+(2-i r \omega) \phi^{\prime \prime}(r)\right)\right)+\omega \phi(r \omega+(-1+i))(r \omega+(1+i))\right)}{2 \sqrt{2} \sqrt{l(l+1)} r^{2} \sqrt{\Gamma(l)}} \\
\Psi_{2}= & -\frac{i e^{-i 2 \varphi}\left(r^{2} \phi^{\prime \prime}+\phi\left(r^{2} \omega^{2}-2\right)\right)\left(\sqrt{\Gamma(l)} \sqrt{\Gamma(l+3)} Y_{l}^{2}+2 e^{i \varphi} \cot (\theta) \sqrt{\Gamma(l-1)} \sqrt{\Gamma(l+2)} Y_{l}^{1}\right)}{4 \sqrt{l(l+1)} r^{3} \sqrt{\Gamma(l-1)} \sqrt{\Gamma(l)}} \\
\Psi_{3}= & \frac{\sqrt{\Gamma(l+2)} e^{-i \varphi} Y_{l}^{1}\left(i\left(\left(r^{2} \omega^{2}-2\right) \phi^{\prime}+r\left(r \phi^{\prime \prime \prime}+(2+i r \omega) \phi^{\prime \prime}\right)\right)+w \phi(2+r \omega(-r \omega+2 i))\right)}{4 \sqrt{2} \sqrt{l(l+1)} r^{2} \sqrt{\Gamma(l)}} \\
\Psi_{4}= & \frac{\sqrt{\Gamma(l+3)} e^{-i 2 \varphi} Y_{l}^{2}\left(i r \phi^{\prime \prime}+(-2 r \omega+2 i) \phi^{\prime}+\omega \phi(-2-i r \omega)\right)}{8 \sqrt{l(l+1)} r^{2} \sqrt{\Gamma(l-1)}} .
\end{aligned}
$$

Open Access. This article is distributed under the terms of the Creative Commons Attribution License (CC-BY 4.0), which permits any use, distribution and reproduction in any medium, provided the original author(s) and source are credited.

\section{References}

[1] C. Liu and D.A. Lowe, Conformal wave expansions for flat space amplitudes, JHEP 07 (2021) 102 [arXiv : 2105.01026] [inSPIRE].

[2] S. Pasterski, S.-H. Shao and A. Strominger, Flat space amplitudes and conformal symmetry of the celestial sphere, Phys. Rev. D 96 (2017) 065026 [arXiv:1701.00049] [InSPIRE].

[3] R.P. Bernar, L.C.B. Crispino and A. Higuchi, Infrared-finite graviton two-point function in static de Sitter space, Phys. Rev. D 90 (2014) 024045 [arXiv:1405.3827] [InSPIRE].

[4] T. Regge and J.A. Wheeler, Stability of a Schwarzschild singularity, Phys. Rev. 108 (1957) 1063 [INSPIRE].

[5] E. Newman and R. Penrose, An approach to gravitational radiation by a method of spin coefficients, J. Math. Phys. 3 (1962) 566 [inSPIRE].

[6] C.S. Meijer, Über eine Erweiterung der Laplace-Transformation I (in German), Proc. Koninkl. Ned. Akad. Wet. 43 (1940) 599.

[7] C.S. Meijer, Über eine Erweiterung der Laplace-Transformation II, Proc. Koninkl. Ned. Akad. Wet. 43 (1940) 702.

[8] H. Kodama and A. Ishibashi, A master equation for gravitational perturbations of maximally symmetric black holes in higher dimensions, Prog. Theor. Phys. 110 (2003) 701 [hep-th/0305147] [INSPIRE].

[9] R.P. Bernar, L.C.B. Crispino and A. Higuchi, Graviton two-point function in $3+1$ static de Sitter spacetime, Int. J. Mod. Phys. D 25 (2016) 1641016 [inSPIRE].

[10] F.J. Zerilli, Effective potential for even parity Regge-Wheeler gravitational perturbation equations, Phys. Rev. Lett. 24 (1970) 737 [INSPIRE]. 
[11] H. Bondi, M.G.J.V. der Burg and A.W.K. Metzner, Gravitational waves in general relativity, VII. Waves from axi-symmetric isolated system, Proc. Roy. Soc. Lond. A 269 (1962) 21.

[12] R.K. Sachs and H. Bondi, Gravitational waves in general relativity VIII. Waves in asymptotically flat space-time, Proc. Roy. Soc. Lond. A 270 (1962) 103.

[13] M. Campanelli, B.J. Kelly and C.O. Lousto, The Lazarus project. II. Space-like extraction with the quasi-Kinnersley tetrad, Phys. Rev. D 73 (2006) 064005 [gr-qc/0510122] [INSPIRE].

[14] A. Nerozzi, Spin coefficients and gauge fixing in the Newman-Penrose formalism, Phys. Rev. D 95 (2017) 064012 [arXiv: 1609.04037] [INSPIRE].

[15] F. de Felice and C.J.S. Clarke, Relativity on curved manifolds, Cambridge University Press, Cambridge, U.K. (1992).

[16] W. Kinnersley, Type D vacuum metrics, J. Math. Phys. 10 (1969) 1195 [InSPIRE].

[17] A. Knapp, Representation theory of semisimple groups: an overview based on examples, Princeton University Press, Princeton, NJ, U.S.A. (2001).

[18] R.P. Boas, Generalized Laplace integrals, Bull. Amer. Math. Soc. 48 (1942) 286.

[19] R.P. Boas, Inversion of a generalized Laplace integral, Proc. Natl. Acad. Sci. 28 (1942) 21.

[20] B.M. Project, H. Bateman, A. Erdélyi and U.S. Office of Naval Research, Tables of integral transforms: based, in part, on notes left by Harry Bateman, volume 1, McGraw-Hill, U.S.A. (1954).

[21] D.A. Lowe and D.M. Ramirez, BMS symmetry via AdS/CFT, JHEP 01 (2021) 075 [arXiv:2007.02851] [INSPIRE]. 